\title{
Practical Perioperative Transoesophageal Echocardiography, 3rd Edition
}

\author{
David Sidebotham, Alan F. Merry, Malcolm E. Legget, I. Gavin Wright (Editors). Oxford \\ University Press, 2018, paperback, 297 pages; ISBN: 9780198759089
}

\author{
Mullein D. Thorleifson, MD (1)
}

Received: 15 January 2019/Accepted: 15 January 2019/Published online: 12 February 2019

(C) Canadian Anesthesiologists' Society 2019

The third edition of Practical Perioperative Transoesophageal Echocardiography, for all intents and purposes, should be considered the current "gold standard" transesophageal echocardiography (TEE) handbook. This edition is concise although not pocket sized - but comprehensive and easily readable. It is not just a brief update but a complete reimagining of its former editions. In addition to the updating of TEE content, the formatting is more readable and appealing to the eye, making it a most enjoyable read. This Third Edition is now published by Oxford University Press as part of the Oxford Clinical Imaging Series. As the series also includes both critical-care echocardiography and point-of-care ultrasonography, it frees up this edition to focus specifically on perioperative TEE.

There are several notable differences in the updated edition. For example, what was once two chapters covering ultrasonography physics and Doppler principles early in the contents is now three sequential chapters with a further, later chapter covering quantitation (seemingly almost an afterthought). This arrangement makes for a more descriptive approach to the physical principles, knobology, artifacts, image quality optimization, and quantitative Doppler imaging. The third chapter contains all the equations a typical echocardiographer would use, with clear directions of when to use them. It also describes those that commercial TEE software systems auto-calculate.

Chapter 4 describes the standard views and how to acquire them (e.g., in which direction to rotate the probe). etc.) are found in chapter 4 . Those new to TEE and just starting to study TEE image acquisition would find it particularly useful to read this section first. These views are

M. D. Thorleifson, MD ( $₫)$

University of Manitoba, Winnipeg, MB, Canada

e-mail: mulleint@gmail.com now described in the same order as the 2013 American Society of Echocardiography Guidelines for Performing a Comprehensive Transesophageal Examination, which provides reassuring consistency across these references. ${ }^{1}$

The left ventricle is particularly well described, as it was in previous editions, but with some improved use of TEE images along with the text. In line with the increased clinical use of three-dimensional (3D) imaging for nearly all structures, the new edition has incorporated 3D imaging into each anatomical section, rather than addressing it in its own, stand-alone chapter.

The chapter on epicardial and epiaortic scanning did not make it into this third edition, nor have the chapters on lung ultrasonography or transthoracic imaging. These omissions are in keeping with the new book's specific focus on TEE. There are new chapters, however, covering mitral and aortic valve repair. Both chapters do an excellent job of covering basic pathological anatomy, patient selection, and mechanisms of repair including offering a how-to guide for acquiring pre- and post-valve repair images. Two other new chapters describe the use of TEE for interventional cardiology procedures and examining the pericardium.

The largest addition to this edition, however, is not in the paper copy per se but in the wealth of online images, video loops, and multiple-choice questions; access to the images and videos requires registration at Oxford Medicine online. Unfortunately, access to the multiple-choice questions is somewhat inconveniently located at a separate location, at Oxford Medicine E-Learning, which requires a separate login and registration. The extensive video library is comprehensive, and the selected images are clear. One production limitation, however, is that as each loop cycles, there is a rather awkward pause, with a blank screen appearing as each clip re-loops. Hence, it cannot be viewed as a typical continuously looped video. 
A further limitation - shared with most print textbooks is that it suffers from the inability to be immediately updated. For example, the Sapien-3 valve is not discussed in the section on transcutaneous valve replacement nor are newer mechanical circulatory devices.

The book particularly shines when it offers images and suggestions that make day-to-day imaging simpler. For example, the mitral valve as clock face images in Chapter 13 offer an orientation of the mitral valve in relation to other cardiac structures in various twodimensional (2D) orientations for localization of paravalvular leaks. The illustrations are clear, well annotated, and practical. Even beginners will be able to appreciate the anatomy, which is frequently illustrated and then shown in 2D and 3D still images.

For what is an otherwise fairly concise text, it is surprisingly and usefully specific. For example, in Chapter 20, the text offers a thorough description of TEE guidance for placing an intra-aortic balloon counterpulsation device that is both pragmatic and clear. Throughout the text, descriptions of normal anatomy and velocities include specific page numbers and locations within the text.

I recommend Practical Perioperative Transoesophageal Echocardiography, $3^{\text {rd }}$ Edition to all experienced echocardiographers who are looking for a handy reference guide for anatomy, pathology, and placement of prostheses and devices. It is also a particularly useful reference for anyone teaching TEE to relative neophytes. Indeed, those studying for echocardiography examinations will find the text comprehensive and enjoyable to read, and the multiple-choice questions, images, and video bank are incredibly valuable.

Conflicts of interest None declared.

Editorial responsibility This submission was handled by Dr. Hilary P. Grocott, Editor-in-Chief, Canadian Journal of Anesthesia.

\section{Reference}

1. Hahn RT, Abraham T, Adams MS, et al. Guidelines for performing a comprehensive transesophageal echocardiographic examination: recommendations from the American Society of Echocardiography and the Society of Cardiovascular Anesthesiologists. J Am Soc Echocardiogr 2013; 26: 921-64.

Publisher's Note Springer Nature remains neutral with regard to jurisdictional claims in published maps and institutional affiliations. 